\title{
Global existence for the regularized surface Quasi-geostrophic equation and its inviscid limits
}

Linrui Li ${ }^{*}$ and Shu Wang

* Correspondence: linrui020213@163.com College of Applied Sciences, Beijing University of Technology, Ping Le Yuan 100, Chaoyang District, Beijing 100124, People's Republic of China

\begin{abstract}
We study solutions of the initial value problem for the 2D regularized surface quasigeostrophic (RSQG) equation. For $\dot{H}^{1}(\Omega) \cap L^{q}(\Omega)(q<2<\infty)$ initial data, we prove the global existence and uniqueness of weak solution for RSQG equation with subcritical powers. For RSQG equation, we establish some regularization results and prove the inviscid limit of the RSQG equation to the classical quasi-geostrophic equation.
\end{abstract}

Mathematics Subject Classifications: 35Q35; 76U05; 86A10.

Keywords: regularized surface quasi-geostrophic equation, initial value problem, existence, uniqueness, regularization

\section{Introduction}

The quasi-geostrophic equation (QG) with periodic boundary conditions on a basic period box $\Omega=[0,2 \pi]^{2} \subset \mathbb{R}^{2}$ is

$$
\left\{\begin{array}{l}
\theta_{t}+\operatorname{div}(u \theta)=0 \\
(-\Delta)^{\frac{1}{2}} \varphi=\theta \\
\nabla^{\perp} \varphi=u \\
\int_{\Omega} \theta \mathrm{dx}=0, \int_{\Omega} \varphi \mathrm{dx}=0, \int_{\Omega} u \mathrm{dx}=0 \\
\theta(x, 0)=\theta_{0}(x) .
\end{array}\right.
$$

where $\theta(x, t)$ is a real-valued function of $x$ and $t$, which represents the potential temperature, and $u$ represents the incompressible horizontal velocity at the surface. The advective velocity $u$ in these equations is determined from $\theta$ by a stream function $\phi$ via the auxiliary relations

$$
u=\left(u_{1}, u_{2}\right)=\left(-\frac{\partial \varphi}{\partial x_{2}}, \frac{\partial \varphi}{\partial x_{1}}\right)
$$

and the relationship $(1.1)_{2}$. The equality relating $u$ to $\theta$ in (1.2) and $(1.1)_{2}$ can be reformulated in terms of periodic Riesz transforms

$$
u=\left(-\partial_{x_{2}} \Lambda^{-1} \theta, \partial_{x_{1}} \Lambda^{-1} \theta\right)=\left(-\mathcal{R}_{2} \theta, \mathcal{R}_{1} \theta\right)
$$


where $\mathcal{R}_{j}, j=1,2$, denotes the Riesz transforms defined by Fourier transform: $\Lambda \equiv(-\Delta)^{\frac{1}{2}}, \Lambda \equiv(-\Delta)^{\frac{1}{2}}$ is the pseudo-differential operator defined in the Fourier space by $\widehat{(-\Delta) \frac{1}{2}} u(k)=|k| \hat{u}(k)$, here $\Delta=\frac{\partial^{2}}{\partial x^{2}}+\frac{\partial^{2}}{\partial y^{2}}$ is the horizontal Laplacian operator. We also write down the regularized surface quasi-geostrophic equation

$$
\left\{\begin{array}{l}
\partial_{t} \theta+\operatorname{div}(u \theta)+\kappa(-\Delta)^{\alpha} \theta_{t}=0, \quad(x, t) \in \Omega \times[0, \infty] \\
u=\left(u_{1}, u_{2}\right)=\left(-\mathcal{R}_{2} \theta, \mathcal{R}_{1} \theta\right), \quad(x, t) \in \Omega \times[0, \infty] \\
\nabla^{\perp} \varphi=u \\
\int_{\Omega} \theta \mathrm{d} x=0, \int_{\Omega} \varphi \mathrm{d} x=0, \int_{\Omega} u \mathrm{~d} x=0 \\
\theta(x, 0)=\theta_{0}(t), \quad x \in \Omega
\end{array}\right.
$$

where $0 \leq \alpha \leq 1$ and $\kappa>0$ are real numbers. This model comes from [1]. The quasigeostrophic equation with dissipative term $\kappa(-\Delta)^{\alpha} \theta$ has received an extensive amount of attentions and has many results in theory and numerical analysis (see e.g., [2-7] for further references), but there are few results on the surface quasi-geostrophic equation with regularized term $\kappa(-\Delta)^{\alpha} \theta_{t}$. Therefore, in this paper, we mainly pay more attention to the regularized equation (1.3) to obtain the global existence, regularity for the solution and the inviscid limit of (1.3). The key issue is still whether weak solutions are regular for all the time. It is well known that for $\alpha>\frac{1}{2}$, the so-called subcritical case, the initial value problem with smooth periodic initial data $\theta_{0}$ has a global smooth solution $[1,8]$.

The quasi-geostrophic equation (1.1) is an important model in geophysical fluid dynamics. It is derived in the special case of constant potential vorticity and buoyancy frequency. Indeed, Equation (1.1) is an important example of a 2D active scalar with a specific structure most closely related to the 3D Euler equation (see [3]). The regularized version of (1.1), (1.1) with the dissipative term $\kappa(-\Delta)^{\alpha} \theta$ or the regularized term $\kappa(-\Delta)^{\alpha} \theta_{t}$, is the dimensionally correct analogue of the 3D incompressible Navier-Stokes equations when $\alpha=1$. In recent years, the 2D quasi-geostrophic equations with and without the dissipative term have attracted significant attention. For the system (1.1), in the previous works of $\mathrm{Wu}[1,9-12]$, the well-posedness results for initial data $\theta_{0}$ in Lebesgue space $L^{p}$, homogeneous Sobolev space $\dot{L}_{s, p}$, Morry space $M_{p, \lambda}$ and Hölder space $C^{r}$ have been studied. Chae [7] obtained these results for the initial data $\theta_{0}$ which belongs to the Triebel-Lizorkin space $F_{p, q}^{s}$ with $s>1+\frac{2}{p}$ and $p, q \in(1, \infty)$. For the critical case, that is $\alpha>\frac{1}{2}$, this problem was first dealt with by Constantin et al. [13] who showed the global existence in Sobolev space $H^{1}$ under smallness assumption of the $L^{\infty}$-norm of the initial temperature $\theta$, but the uniqueness is proved for initial data $H^{2}$. A. Córdoba and D. Córdoba [14] proved that the viscosity solutions are smooth on the interval $t \leq T_{1}$ and $t \geq T_{2}$. A. Kiselev et al. [5] proved global existence of large smooth solutions. Later, in [1], Wu reformulates the problem as an integral equation and applies the Banach contraction mapping principle to prove local existence with initial value $\theta_{0} \in H^{s}(s>1)$. Another recent progress on the critical dissipative QG equation was given in the work by Caffarelli and Vasseur [15]. The supercritical case $\alpha>\frac{1}{2}$ is open. For more results, see [1,9,16-18] and references 
therein. However, all these results mainly concentrate on the general quasi-geostrophic equation with dissipative term $\kappa(-\Delta)^{\alpha} \theta$.

Recently, Khouider and Titi [6] study the following regularized model of surface quasi-geostrophic equation:

$$
\left\{\begin{array}{l}
\frac{\partial \tilde{\theta}^{\alpha}}{\partial t}+\operatorname{div}\left(u^{\alpha} \theta^{\alpha}\right)=0 \\
(-\Delta)^{\frac{1}{2}} \varphi^{\alpha}=\theta^{\alpha} \\
\left(1-\alpha^{2} \Delta\right) \theta^{\alpha}=\tilde{\theta}^{\alpha} \\
\nabla^{\perp} \psi^{\alpha}=u^{\alpha} \\
\theta^{\alpha}(x, 0)=\theta_{0}(x) .
\end{array}\right.
$$

They showed that the model (1.4) admits a maximum principle and obtained a necessary and sufficient condition that the solution of the regularized QG equations (1.4) develops a singularity in finite time and proves that, if the initial condition is smooth, then the regularized solution remains as smooth as the initial data for all times.

In the present paper, we will study the model (1.3) in the subcritical $\alpha>\frac{1}{2}$ and critical case $\alpha>\frac{1}{2}$. On the one hand, we will establish the solution with lower regularity for initial data $\theta_{0} \in \dot{H}^{1}(\Omega)$, and this improved the global existence for initial data $\theta_{0}$ $\in H^{s}(s>1)$ in [1]. On the other hand, we generalize the results for (1.4) to the generally regularized models (1.3).

The rest of this article is organized as follows. In Section 2, we present the global existence for the regularized Equation (1.3). In Section 3, we give the regularization results for the regularized model (1.3) and obtain the maximum principle. Section 4 is devoted to the inviscid limit from the regularized surface quasi-geostrophic equation to classical surface quasi-geostrophic equation.

\section{Global existence for the regularized surface quasi-geostrophic equation}

In this section, we establish existence and uniqueness of global weak solutions of regularized model (1.3).

Firstly, we rewrite the equation (1.3) $)_{1}$ as a functional differential equation in the form

$$
\left(1+\kappa \Lambda^{2 \alpha}\right) \theta_{t}=-\operatorname{div}(u \theta)
$$

or

$$
\theta_{t}=-\left(1+\kappa \Lambda^{2 \alpha}\right)^{-1} \operatorname{div}(u \theta)
$$

Noticing that if $\theta$ is in the Sobolev space $H^{1}(\Omega)$, then $\psi=(-\Delta)^{-\frac{1}{2}} \theta=\Lambda^{-1} \theta$ belongs to the Sobolev space $H^{2}(\Omega)$. This implies that $u=\nabla^{\perp} \psi$ is in $H^{1}(\Omega)$. Furthermore, we have the following lemma, which is proved in [6].

Lemma 2.1 Let $\theta \in \dot{H}^{1}(\Omega)=\left\{u \in H^{1}(\Omega), \int_{\Omega} u \mathrm{~d} x=0\right\}$ and $u \in \dot{H}^{1}(\Omega) \times \dot{H}^{1}(\Omega)$ then $\operatorname{div}(u \theta) \in \dot{H}^{-1}(\Omega)$, where $\dot{H}^{-1}(\Omega)$ is the dual space of $\dot{H}^{1}(\Omega)$. Moreover, for $u \in \dot{H}^{1}(\Omega) \times \dot{H}^{1}(\Omega)$ fixed, $\theta \rightarrow \operatorname{div}(\theta u)$ is a linear continuous operator from $\dot{H}^{1}(\Omega)$ to $\dot{H}^{-1}(\Omega)$.

This lemma immediately yields the following corollary: 
Corollary 2.2 Let $u \in \dot{H}^{1}(\Omega) \times \dot{H}^{1}(\Omega)$ satisfying $\operatorname{div} u=0$ and $\theta \in \dot{H}^{1}(\Omega)$, then $<\operatorname{div}$ $(u \theta), \theta>=0$.

Now, we state and prove the global existence result for weak solutions for all time if the initial condition $\theta_{0}$ belongs to $\dot{H}^{1}(\Omega)$. More precisely, we have the following theorem.

Theorem 2.3 Let $\alpha>\frac{1}{2}$ and $\theta_{0} \in \dot{H}^{1}(\Omega) \cap L^{q}(\Omega), 2<q<\infty$, then the initial value problem (1.3) has a global unique solution $\theta \in C\left((0,+\infty), \dot{H}^{1}(\Omega)\right)$.

Proof. Due to (1.3) and (2.2), we can have

$$
\theta_{t}=-\left(1+\kappa \Lambda^{2 \alpha}\right)^{-1} \operatorname{div}(u \theta) \equiv F(\theta) .
$$

Therefore, by Lemma 2.1, we know that $\operatorname{div}(u \theta) \in \dot{H}^{-1}(\Omega)$ (i.e., $\theta_{t} \in \dot{H}^{-1}(\Omega)$ ). We first prove local existence and uniqueness. For this, it is enough to establish that the function $\operatorname{div}(u \theta)$ is locally Lipshitz as a map from $\dot{H}^{-1}(\Omega)$ into $\dot{H}^{-1}(\Omega)$. Before proving the locally Lipshitz condition, we give some important inequality we will use.

Taking $\phi \in \dot{H}^{1}(\Omega)$, then

$$
\begin{aligned}
|<\operatorname{div}(u \theta), \phi>| & =\left|\int_{\Omega} \theta(x) u(x) \cdot \nabla \phi(x) \mathrm{d} x\right| \\
& \leq C\|u\|_{L^{4}(\Omega)}\|\theta\|_{L^{4}(\Omega)}\|\nabla \phi\|_{L^{2}(\Omega)} \\
& \leq C\|u\|_{L^{2}(\Omega)}^{\frac{1}{2}}\|\nabla u\|_{L^{2}(\Omega)}^{\frac{1}{2}}\|\theta\|_{L^{2}(\Omega)}^{\frac{1}{2}}\|\nabla \theta\|_{L^{2}(\Omega)}^{\frac{1}{2}}\|\nabla \phi\|_{L^{2}(\Omega)},
\end{aligned}
$$

by the 2D Gagliardo-Nirenberg-Ladyzhenskaya interpolation inequality, we can obtain

$$
\|\operatorname{div}(u \theta)\|_{H^{-1}} \leq C\|u\|_{L^{2}(\Omega)}^{\frac{1}{2}}\|\nabla u\|_{L^{2}(\Omega)}^{\frac{1}{2}}\|\theta\|_{L^{2}(\Omega)}^{\frac{1}{2}}\|\nabla \theta\|_{L^{2}(\Omega)}^{\frac{1}{2}} \leq\|\nabla \theta\|_{L^{2}}^{\frac{1}{2}}
$$

which implies that $\operatorname{div}(u \theta)$ is bounded in $\dot{H}^{-1}(\Omega)$ by using lemma 2.1 .

Next, we use the inequality (2.4) to show the $\operatorname{div}(u \theta)$ is locally Lipschitz

$$
\begin{aligned}
\left\|F\left(\theta_{1}\right)-F\left(\theta_{2}\right)\right\|_{H^{-1}}= & \left\|\left(1+\kappa \Lambda^{2 \alpha}\right)^{-1}\left(\operatorname{div}\left(\theta_{1} u_{1}\right)-\operatorname{div}\left(\theta_{2} u_{2}\right)\right)\right\|_{H^{-1}} \\
\leq & \left\|\left(1+\kappa \Lambda^{2 \alpha}\right)^{-1}\right\|_{L^{2}}\left(\left\|\operatorname{div}\left(u_{1}\left(\theta_{1}-\theta_{2}\right)\right)\right\|_{H^{-1}}\right. \\
& \left.+\left\|\operatorname{div}\left(u_{1}-u_{2}\right) \theta_{2}\right\|_{H^{-1}}\right) \\
\leq & C\left(\left\|u_{1}\right\|_{L^{2}}^{\frac{1}{2}}\left\|\nabla u_{1}\right\|_{L^{2}}^{\frac{1}{2}}\left\|\theta_{1}-\theta_{2}\right\|_{L^{2}}^{\frac{1}{2}}\left\|\nabla\left(\theta_{1}-\theta_{2}\right)\right\|_{L^{2}}^{\frac{1}{2}}\right. \\
& \left.+\left\|u_{1}-u_{2}\right\|_{L^{2}}^{\frac{1}{2}}\left\|\nabla\left(u_{1}-u_{2}\right)\right\|_{L^{2}}^{\frac{1}{2}}\left\|\theta_{2}\right\|_{L^{2}}^{\frac{1}{2}}\left\|\nabla \theta_{2}\right\|_{L^{2}}^{\frac{1}{2}}\right),
\end{aligned}
$$

where we have used the boundedness of Riesz transforms in $\dot{H}^{-1}$ space. Then, using the Poincaré inequality

$$
\|u\|_{L^{2}} \leq C\|\nabla u\|_{L^{2}}, \quad \forall u \in \dot{H}^{1}(\Omega),
$$

we have

$$
\begin{aligned}
\left\|F\left(\theta_{1}\right)-F\left(\theta_{2}\right)\right\|_{H^{-1}} \leq & C\left(\left\|\nabla u_{1}\right\|_{L^{2}}\left\|\nabla\left(\theta_{1}-\theta_{2}\right)\right\|_{L^{2}}\right. \\
& \left.+\left\|\nabla\left(u_{1}-u_{2}\right)\right\|_{L^{2}}\left\|\nabla \theta_{2}\right\|_{L^{2}}^{\frac{1}{2}}\right) .
\end{aligned}
$$


On the other hand, using the facts that the functional operator $\theta \rightarrow u=\nabla^{\perp}\left[(-\Delta)^{-\frac{1}{2}} \theta\right]$ is an isomorphism from $\dot{H}^{1}$ into $\dot{H}^{1} \times \dot{H}^{-1}$ and $\theta \rightarrow(1+$ $\left.\kappa \Lambda^{2 \alpha}\right)^{-1} \theta_{t}$ is a bounded operator from $\dot{H}^{1}$ into $\dot{H}^{-1}$, and using the Poincaré inequality (2.5), we know that the following norm is equivalent

$$
\|\nabla u\|_{L^{2}} \sim\|\nabla \theta\|_{L^{2}} \sim\|\theta\|_{\dot{H}^{1}} .
$$

Therefore,

$$
\left\|F\left(\theta_{1}\right)-F\left(\theta_{2}\right)\right\|_{\dot{H}^{-1}} \leq C\left(\left\|\theta_{1}-\theta_{2}\right\|_{\dot{H}^{-1}}\right)\left(\left\|\theta_{1}\right\|_{\dot{H}^{-1}}+\left\|\theta_{2}\right\|_{\dot{H}^{-1}}\right) .
$$

Thus, by the fixed point theory, we have the short time existence and uniqueness of solution for the functional differential equation (2.3).

Suppose that $\left[0, T^{*}\right]$ is the maximal interval of existence of the solution $\theta \in C\left(\left[0, T^{*}\right], \dot{H}^{1}(\Omega)\right)$.

Now, we show the global existence for (1.3). To do this, it suffices to prove that the norm $\|\theta\|_{\dot{H}^{1}}$ stays bounded on the maximal interval of existence for the solution $\theta$ of the regularized surface quasi-geostrophic equation (1.3) in the subcritical case $\alpha>\frac{1}{2}$.

Let $\theta \in C\left([0, T] ; \dot{H}^{1}(\Omega)\right)$ be the solution of the initial value problem (1.3). Take the inner produce of $\Lambda^{2-2 \alpha} \theta$ with the first Equation in (1.3)

$$
\frac{1}{2} \frac{d}{d t}\left(\left\|\Lambda^{1-\alpha} \theta\right\|_{2}^{2}+\kappa\|\Lambda \theta\|_{2}^{2}\right)=-\left(\Lambda^{2-2 \alpha} \theta, u \cdot \nabla \theta\right) .
$$

For the right-hand side of (2.6), we have

$$
\left|\left(\Lambda^{2-2 \alpha} \theta, u \cdot \nabla \theta\right)\right|=\left|\left(\Lambda^{2-2 \alpha} \theta, \nabla(u \theta)\right)\right| \leq\left\|\Lambda^{1+a-\alpha} \theta\right\|_{L^{2}}\left\|\Lambda^{2-a-\alpha}(u \theta)\right\|_{L^{2}},
$$

where $a \leq \alpha$ remains to be determined. By the calculus inequality for the CalderonZygmund type singular integral, we obtain

$$
\left\|\Lambda^{2-a-\alpha}(u \theta)\right\|_{L^{2}} \leq C\left(\left\|\Lambda^{2-a-\alpha} u\right\|_{L^{p}}\|\theta\|_{L^{q}}+\left\|\Lambda^{2-a-\alpha} \theta\right\|_{L^{p}}\|u\|_{L^{q}}\right),
$$

where $\frac{1}{p}+\frac{1}{q}=\frac{1}{2}$ and $2<q<\infty$. Considering the second equation in (1.3), by the Calderon-Zygmund inequalities, we obtain

$$
\|u\|_{L^{q}} \leq C\|\theta\|_{L^{q}}
$$

and

$$
\left\|\Lambda^{2-a-\alpha} u\right\|_{L^{q}} \leq C\left\|\Lambda^{2-a-\alpha} \theta\right\|_{L^{q}},
$$

where $1<q<\infty$.

By the Sobolev imbedding $W^{1+2-\frac{2}{p}-\alpha-a, 2} \subset W^{2-\alpha-a, q}$, then (2.10) becomes

$$
\left\|\Lambda^{2-a-\alpha} u\right\|_{L^{q}} \leq C\left\|\Lambda^{2-a-\alpha} \theta\right\|_{L^{q}} \leq C\left\|\Lambda^{1+2-a-\alpha+\frac{2}{p}} \theta\right\|_{L^{2}} .
$$

Putting (2.11) into (2.8), then (2.7) becomes

$$
\left|\left(\Lambda^{2-2 \alpha} \theta, u \cdot \nabla \theta\right)\right| \leq C\left\|\Lambda^{1+a-\alpha} \theta\right\|_{L^{2}}\|\theta\|_{L^{q}}\left\|\Lambda^{1+2-a-\alpha+\frac{2}{p}} \theta\right\|_{L^{2}} .
$$


In the above analysis, $a$ is essentially arbitrary and we can choose $a=1+\frac{1}{p}$ without loss of generality so that $1+a-\alpha=1+2-\alpha+\frac{2}{p}-a$. Therefore, we get

$$
\left|\left(\Lambda^{2-2 \alpha} \theta, u \cdot \nabla \theta\right)\right| \leq C\|\theta\|_{L^{q}}\left\|\Lambda^{1+a-\alpha} \theta\right\|_{L^{2}}^{2} .
$$

Putting (2.13) into (2.6), we have

$$
\begin{aligned}
& \frac{1}{2} \frac{d}{d t}\left(\left\|\Lambda^{1-\alpha} \theta\right\|_{L^{2}}^{2}+\kappa\|\Lambda \theta\|_{L^{2}}^{2}\right) \\
& \quad \leq C\|\theta\|_{L^{q}}\left\|\Lambda^{1+a-\alpha} \theta\right\|_{L^{2}}^{2} \\
& \quad \leq C\|\theta\|_{L^{q}}\left\|\Lambda^{1-\alpha} \theta\right\|_{L^{2}}^{\frac{a}{\alpha}}\|\Lambda \theta\|_{L^{2}}^{1-\frac{a}{\alpha}} \\
& \quad \leq C\left\|\theta_{0}\right\|_{L^{q}}\left(\frac{C}{\kappa}\left\|\Lambda^{1-\alpha} \theta\right\|_{L^{2}}^{2}+\frac{\kappa}{C}\|\Lambda \theta\|_{L^{2}}^{2}\right),
\end{aligned}
$$

where we have used the inequality $\|\theta\|_{L^{q}} \leq\left\|\theta_{0}\right\|_{L^{q}}, 2<q<\infty, 2<q<\infty$, it is easy to prove for the regularized surface quasi-geostrophic equation. By the inequality (2.14), we obtain

$$
\frac{1}{2} \frac{\mathrm{d}}{\mathrm{d} t}\left(\left\|\Lambda^{1-\alpha} \theta\right\|_{L^{2}}^{2}+\kappa\|\Lambda \theta\|_{L^{2}}^{2}\right) \leq C\left(\left\|\Lambda^{1-\alpha} \theta\right\|_{L^{2}}^{2}+\kappa\|\Lambda \theta\|_{L^{2}}^{2}\right) .
$$

Thanks to the Gronwall's lemma, we obtain

$$
\left\|\Lambda^{1-\alpha} \theta\right\|_{L^{2}}^{2}+\kappa\|\Lambda \theta\|_{L^{2}}^{2} \leq\left(\left\|\Lambda^{1-\alpha} \theta_{0}\right\|_{L^{2}}^{2}+\kappa\left\|\Lambda \theta_{0}\right\|_{L^{2}}^{2}\right) e^{\frac{C}{2} t} .
$$

This guarantees that the $\dot{H}^{1}$ norm of $\theta$ is bounded. Therefore, the local solution can be extended uniquely to $\left[0,2 T_{0}\right]$ and the global solution is obtained by repeating this procedure. This completes the proof of Theorem 2.1.

\section{Regularity results}

In this section, we investigate the higher regularity and prove the maximum principle for the regularized system (1.3).

Theorem 3.1 (Regularity) Let $\theta_{0} \in \dot{H}^{m}(\Omega), m \geq 1, \alpha=1$, then the solution for the regularized problem of quasi-geostrophic equation exists a solution $\theta(t) \in C\left((0,+\infty), \dot{H}^{m}\right)$.

Proof. The case $m=1$ follows from Theorem 2.1. The case $m>1$ had been obtained by $\mathrm{Wu}$ in [1]. For completeness, here we give a different proof by following the proof. We need to proceed the steps by induction.

It is obvious that if $m \geq 2$ then $\theta \in \dot{H}^{m}$ equivalent to $u \in \dot{H}^{m} \times \dot{H}^{m}$, which hints that $\operatorname{div}(u \theta) \in \dot{H}^{m-2}$. By applying the Gagliardo-Nirenberg-Ladyzhenskaya interpolation inequality, as in Theorem 2.1, we have

$$
\begin{aligned}
\int_{\Omega}\left|D^{m-2} \operatorname{div}(u \theta)\right|^{2} \mathrm{~d} x & =\int_{\Omega}\left|D^{m-2}(u \cdot \nabla \theta)\right|^{2} \mathrm{~d} x \\
\leq & C \sum_{k=0}^{m-2} \int_{\Omega}\left|D^{k} u \cdot \nabla D^{m-2-k} \theta\right|^{2} \mathrm{~d} x \\
\leq & C \sum_{k=0}^{m-2}\left\|D^{k} u\right\|_{L^{4}(\Omega)}^{2}\left\|\nabla D^{m-k-2} \theta\right\|_{L^{4}(\Omega)}^{2} \\
\leq & C \sum_{k=0}^{m-2}\left\|D^{k} u\right\|_{L^{2}(\Omega)}\left\|\nabla D^{k} u\right\|_{L^{2}(\Omega)} \\
& \times\left\|\nabla D^{m-k-2} \theta\right\|_{L^{2}(\Omega)}\left\|\nabla \nabla D^{m-k-2} u\right\|_{L^{2}(\Omega)} \\
\leq & C\|u\|_{H^{m}(\Omega)}^{2}\|\theta\|_{H^{m}(\Omega)}
\end{aligned}
$$


In addition, similar steps as in the proof of Theorem 2.1 yield

$$
\begin{aligned}
\| F & \left(\theta_{1}\right)-F\left(\theta_{2}\right) \|_{\dot{H}^{m-2}} \\
= & \left\|\left(1+\kappa \Lambda^{2 \alpha}\right)^{-1}\left(\operatorname{div}\left(\theta_{1} u_{1}\right)-\operatorname{div}\left(\theta_{2} u_{2}\right)\right)\right\|_{\dot{H}^{m-2}} \\
\leq & \left\|\left(1+\kappa \Lambda^{2 \alpha}\right)^{-1}\right\|\left(\left\|\operatorname{div}\left(u_{1}\left(\theta_{1}-\theta_{2}\right)\right)\right\|_{\dot{H}^{m-2}}\right. \\
& \left.+\left\|\operatorname{div}\left(u_{1}-u_{2}\right) \theta_{2}\right\|_{\dot{H}^{m-2}}\right) \\
\leq & C\left(\left\|u_{1}\right\|_{\dot{H}^{m}}\left\|\theta_{1}-\theta_{2}\right\|_{\dot{H}^{m-2}}\right. \\
& +\left\|u_{1}-u_{2}\right\|_{\dot{H}^{m}}\left\|\theta_{2}\right\|_{\dot{H}^{m}}
\end{aligned}
$$

This proves the local existence and uniqueness of smooth solutions for the (1.3) in $\dot{H}^{m-2}$.

Next, we will show that the $\theta(t)$ is bounded in $\dot{H}^{m}(\Omega)$. To prove the global existence, i.e., it suffices to prove that $\left\|D^{m-1} \theta(t)\right\|_{L^{2}}^{2}+\left\|\nabla D^{m-1} \theta(t)\right\|_{L^{2}}^{2}$ remains bounded in any finite interval of time.

The case $m=1$ is proved in Theorem 2.3. Assume by induction that $\theta \in C\left((0,+\infty), H^{m-1} \cap \dot{H}^{-1}\right)$. If $\theta \in \dot{H}^{m} \cap \dot{H}^{1}$, then $D^{m-1} \theta \in \dot{H}^{1}$, thus $\Delta D^{m-1} \theta \in \dot{H}^{1}$ and we can obtain

$$
<\left(D^{m-1} \theta_{t}+\kappa(-\Delta) D^{m-1} \theta_{t}\right), D^{m-1} \theta>=-<D^{m-1}(\operatorname{div}(u \theta)), D^{m-1} \theta>,
$$

Let $\phi(t)=\left\|D^{m-1} \theta(t)\right\|_{L^{2}}^{2}+\left\|\nabla D^{m-1} \theta(t)\right\|_{L^{2}}^{2}$, we have

$$
\begin{aligned}
\frac{\mathrm{d}}{\mathrm{d} t} \phi(t) & \\
& =-\int_{\Omega} \sum_{k=0}^{m-1} C_{k}^{m-1} D^{k} u \cdot \nabla\left(D^{m-k-1} \theta\right) D^{m-1} \theta \mathrm{d} x \\
& =-\int_{\Omega} \sum_{k=0}^{m-2} C_{k}^{m-1} D^{k} u \cdot \nabla\left(D^{m-k-1} \theta\right) D^{m-1} \theta \mathrm{d} x+\int_{\Omega} u \cdot \nabla\left(D^{m-1} \theta\right) D^{m-1} \theta \mathrm{d} x \\
& =-\int_{\Omega} \sum_{k=0}^{m-2} C_{k}^{m-1} D^{k} u \cdot \nabla\left(D^{m-k-1} \theta\right) D^{m-1} \mathrm{~d} x+\frac{1}{2} \int_{\Omega} \operatorname{div}\left(u\left(D^{m-1} \theta\right)^{2}\right) \mathrm{d} x \\
& =-\int_{\Omega}^{m-2} \sum_{k=0}^{m-2} C_{k}^{m-1} D^{k} u \cdot \nabla\left(D^{m-k-1} \theta\right) D^{m-1} \theta \mathrm{d} x \\
& \leq C \sum_{k=1}^{m-2}\left\|D^{k} u\right\|_{L^{2}}\left\|D^{m-1-k} \nabla \theta\right\|_{L^{4}}\left\|D^{m-1} \theta\right\|_{L^{4}} \\
& \leq C \sum_{k=1}^{m-2}\|u\|_{H^{k}}\left\|D^{m-1-k} \nabla \theta\right\|_{L^{2}}^{\frac{1}{2}}\left\|\nabla D^{m-1-k} \nabla \theta\right\|_{L^{2}}^{\frac{1}{2}}\left\|D^{m-1} \theta\right\|_{L^{2}}^{\frac{1}{2}}\left\|\nabla D^{m-1} \theta\right\|_{L^{2}}^{\frac{1}{2}} \\
& \leq C\left(\sum_{k=1}^{m-2}\|u\|_{H^{k}}\right)\|\theta\|_{H^{m-1}}\|\nabla \theta\|_{H^{m-1}} \\
& \leq C(\alpha)\left(\sum_{k=1}^{m-2}\|u\|_{H^{k}}\right) \phi(t)=\psi(t) \phi(t),
\end{aligned}
$$


where $\psi(t)=C(\alpha)\left(\sum_{k=1}^{m-2}\|u\|_{H^{k}}\right)$. . Thus, by the Gronwall's lemma we obtain,

$$
\phi(t) \leq \phi(0) \exp \left(\int_{0}^{t} \psi(s) \mathrm{d} s\right)
$$

Thanks to that $\psi(t)$ is bounded by the induction assumption, we get $\varphi(t)$ is bounded on any finite interval of time. This completes the proof of Theorem 3.1.

Then, we will extend the maximum principle in $[14,17]$ to the regularized surface quasi-geostrophic equation by using the method of Khouider and Titi [6].

Theorem 3.2 Let $\theta_{0} \in \dot{H}^{1}(\Omega) \cap L^{\infty}(\Omega)$ and $\alpha=1, \kappa$ is positive number, then the solution $\theta$ of the regularized problem (1.3) satisfies

$$
\|\theta(t)\|_{L^{\infty}} \leq\left\|\theta_{0}\right\|_{L^{\infty}}, \quad \forall \quad t \geq 0 .
$$

Moreover, if $\theta_{0}(x) \geq 0, \forall x \in \Omega$, then

$$
\theta(x, t) \geq 0, \quad t>0, \quad x \in \Omega .
$$

Proof. Let $\theta(x, t)$ be the solution of (1.3), then $\left(\theta+\left\|\theta_{0}\right\|_{L^{\infty}}\right)$ satisfies

$$
\frac{\partial}{\partial t}\left(\theta+\left\|\theta_{0}\right\|_{L^{\infty}}\right)+\kappa(-\Delta) \frac{\partial}{\partial t}\left(\theta+\left\|\theta_{0}\right\|_{L^{\infty}}\right)+u \cdot \nabla\left(\theta+\left\|\theta_{0}\right\|_{L^{\infty}}\right)=0 .
$$

Denote $u_{-}=\max \{-u, 0\}$, if $u \in \dot{H}^{1}(\Omega)$ then $u_{-} \in \dot{H}^{1}(\Omega)$, we have

$$
\nabla u_{-}= \begin{cases}\nabla u, & \text { if } u<0 \\ 0, & \text { if } u \geq 0\end{cases}
$$

Multiplying the above equation (3.2) by $\left(\theta+\left\|\theta_{0}\right\|_{L^{\infty}}\right)_{-}$, we have

$$
\frac{1}{2} \frac{\mathrm{d}}{\mathrm{d} t}\left(\int_{\Omega}\left(\theta+\left\|\theta_{0}\right\|_{L^{\infty}}\right)_{-}^{2} \mathrm{~d} x+k \int_{\Omega}\left|\nabla\left(\theta+\left\|\theta_{0}\right\|_{L^{\infty}}\right)_{-}\right|^{2} \mathrm{~d} x\right)=0,
$$

which yields

$$
\begin{aligned}
& \left\|\left(\theta+\left\|\theta_{0}\right\|_{L^{\infty}}\right)-\right\|_{L^{2}}+k\left\|\nabla\left(\theta+\left\|\theta_{0}\right\|_{L^{\infty}}\right)_{-}\right\|_{L^{2}}^{2} \\
& =\left\|\left(\theta(0)+\left\|\theta_{0}\right\|_{L^{\infty}}\right)-\right\|_{L^{2}}+k\left\|\nabla\left(\theta_{0}+\left\|\theta_{0}\right\|_{L^{\infty}}\right)_{-}\right\|_{L^{2}}^{2},
\end{aligned}
$$

It is obvious that the right-hand side of (3.4) is zero, by the fact

$$
\left(\theta_{0}+\left\|\theta_{0}\right\|_{L^{\infty}}\right)_{-} \equiv 0,
$$

Therefore,

$$
\left(\theta+\left\|\theta_{0}\right\|_{L^{\infty}}\right)_{-} \equiv 0 .
$$

which implies

$$
\theta(x, t) \geq-\left\|\theta_{0}\right\|_{L^{\infty}} .
$$

Similarly, we have

$$
\theta(x, t) \leq\left\|\theta_{0}\right\|_{L^{\infty}} .
$$


Hence (3.1) holds.

Next, we show the rest of Theorem 3.2. Assume $\theta_{0}(x) \geq 0, x \in \Omega$. Multiplying the evolution equation for $\theta$ by $\theta_{-}=\max \{-\theta, 0\}$ and integrating over the domain in the similar way as above, we have

$$
\frac{1}{2} \frac{\mathrm{d}}{\mathrm{d} t}\left(\int_{\Omega} \theta_{-}^{2} \mathrm{~d} x+k \int_{\Omega}-\nabla\left(\theta_{-}\right)-{ }^{2} \mathrm{~d} x\right)=0,
$$

i.e.,

$$
\left\|\theta_{-}(t)\right\|_{L^{2}}^{2}+k\left\|\nabla \theta_{-}(t)\right\|_{L^{2}}^{2}=\left\|\theta_{-}(0)\right\|_{L^{2}}^{2}+k\left\|\nabla \theta_{-}(0)\right\|_{L^{2}}^{2}=0,
$$

Owing to $\theta_{0} \geq 0$, we have $\theta_{-}(t) \equiv 0, \forall t>0$. Therefore, $\theta(x, t) \geq 0$. This completes the proof of Theorem 3.2.

\section{Inviscid limit}

In this section, we investigate the convergence of the solution of the regularized surface quasi-geostrophic equation (1.3) to a solution of the classical surface quasi-geostrophic equation (1.1) as $\kappa$ tends to zero. We have the following result

Theorem 4.1 Let $\theta_{1}$ and $\theta_{2}$ be the smooth solutions of the RSQG equations (1.3) and the classical QG equations (1.1) with the same initial data $\theta_{0} \in \dot{H}^{\alpha+1}(\Omega)$, defined on the maximal time interval of existence $\left[0, T^{*}\right]$, then for any $t<T^{*}$,

$$
\left\|\theta_{1}(\cdot, t)-\theta_{2}(\cdot, t)\right\|_{L^{2}(\Omega)} \leq C \kappa,
$$

where $C$ is a constant depending only $T^{*}$ and $\left\|\theta_{0}\right\|_{H^{\alpha+1}(\Omega)}$.

Proof. Let $u_{1}$ and $u_{2}$ be the velocity field corresponding $\theta_{1}$ and $\theta_{2}$, respectively. Then, the difference $\theta(x, t)=\theta_{1}(x, t)-\theta_{2}(x, t)$ solves the equation

$$
\theta_{t}+u_{1} \nabla \theta+u \cdot \nabla \theta_{2}+\kappa \Lambda^{2 \alpha} \theta_{t}+\kappa \Lambda^{2 \alpha}\left(\theta_{2}\right)_{t}=0,
$$

where $u=u_{1}-u_{2}$. Multiplying (4.1) by $\theta(x, t)$ and integrating over $\Omega$, we get

$$
\frac{1}{2} \frac{\mathrm{d}}{\mathrm{d} t} \int_{\Omega}\left(\theta^{2}+\kappa\left|\Lambda^{\alpha} \theta\right|^{2}\right) \mathrm{d} x=-\int_{\Omega} u \cdot \nabla \theta_{2} \cdot \theta \mathrm{d} x-\kappa \int_{\Omega} \theta \Lambda^{2 \alpha}\left(\theta_{2}\right)_{t} \mathrm{~d} x,
$$

where the two terms on the right-hand side of (4.2) may be estimated as follows,

$$
-\int_{\Omega} u \cdot \nabla \theta_{2} \cdot \theta d x \leq\left\|\nabla \theta_{2}\right\|_{L^{\infty}}\|u\|_{L^{2}}\|\theta\|_{L^{2}}
$$

Since $\|u\|_{2} \leq C\|\theta\|_{2}$, it follows that,

$$
-\int_{\Omega} u \cdot \nabla \theta_{2} \cdot \theta \mathrm{d} x \leq C\left\|\nabla \theta_{2}(\cdot, t)\right\|_{L^{\infty}}\|\theta\|_{L^{2}}^{2} .
$$

To proceed, we need the calculus inequality for the Calderon-Zygmund type singular integral

$$
\left\|\Lambda^{\gamma}(F G)\right\|_{L^{r}} \leq C\left(\left\|\Lambda^{\gamma} F\right\|_{L^{p}}\|G\|_{L^{q}}+\|F\|_{L^{q}}\left\|\Lambda^{\gamma} G\right\|_{L^{p}}\right),
$$

where $\gamma>0,1<\gamma \leq p \leq \infty$ and $\frac{1}{\gamma}=\frac{1}{p}+\frac{1}{q}$. Noticing the fact that $\left(\theta_{2}\right)_{t}+u_{2} \cdot \nabla \theta_{2}=0$ and applying the inequality (4.3), we can bound the second term of right-hand side of 
(4.2) by

$$
\begin{aligned}
& \kappa\left|\int_{\Omega} \theta \Lambda^{2 \alpha+1}\left(u_{2} \theta_{2}\right) \mathrm{d} x\right| \\
\leq & \left\|\Lambda^{\alpha} \theta\right\|_{L^{2}}^{2}+\frac{\kappa^{2}}{4}\left\|\Lambda^{\alpha+1}\left(u_{2} \theta_{2}\right)\right\|_{L^{2}}^{2} \\
\leq & \left\|\Lambda^{\alpha} \theta\right\|_{L^{2}}^{2}+C \kappa^{2}\left(\left\|\Lambda^{\alpha+1} u_{2}\right\|_{L^{2}}\left\|\theta_{2}\right\|_{L^{\infty}}+\left\|u_{2}\right\|_{L^{\infty}}\left\|\Lambda^{\alpha+1} \theta_{2}\right\|_{L^{2}}\right)^{2} \\
\leq & \left\|\Lambda^{\alpha} \theta\right\|_{L^{2}}^{2}+C \kappa^{2}\left\|\Lambda^{\alpha+1} \theta_{2}\right\|_{L^{2}}^{2}\left\|\theta_{2}\right\|_{H^{\alpha+1}}^{2} \\
\leq & \left\|\Lambda^{\alpha} \theta\right\|_{L^{2}}^{2}+C \kappa^{2}\left\|\theta_{2}\right\|_{H^{\alpha+1}}^{4} .
\end{aligned}
$$

Therefore,

$$
\frac{\mathrm{d}}{\mathrm{d} t} \int_{\Omega}\left(\theta^{2}+\kappa\left|\Lambda^{\alpha} \theta\right|^{2}\right) \mathrm{d} x \leq C\left(\left\|\nabla \theta_{2}(\cdot, t)\right\|_{L^{\infty}} \int_{\Omega} \theta^{2} \mathrm{~d} x+\left\|\Lambda^{\alpha} \theta\right\|_{L^{2}}^{2}\right)+C \kappa^{2}\left\|\theta_{2}\right\|_{H^{\alpha+1}}^{4}
$$

where the constant $C$ does not depend on $\kappa$. By the Gronwall's Lemma, we get the desired result.

\section{Acknowledgements}

This work is supported by NSFC (Grant No. 10771009), BSFC (Grant No. 1085001) of China, Funding Project for Academic Human Resources Development in Institutions of Higher Leading Under the Jurisdiction of Beijing Municipality(PHR-IHLB 200906103) and Beijing Education Committee Funds.

\section{Authors' contributions}

All the authors typed, read, and approved the final manuscript.

\section{Competing interests}

The authors declare that they have no competing interests.

Received: 23 May 2011 Accepted: 27 October 2011 Published: 27 October 2011

\section{References}

1. Wu, J: The Quasi-geostrophic equations and its two regularizations. Communications in Partial Differential Equations. 27(5/6):1161-1181 (2002)

2. Pedlosky, J: Geophysical Fluid Dynamics. Springer, New York (1987)

3. Constantin, P, Majda, A, Tabak, E: Formation of strong fronts in the 2-D quasi-geostrophic thermal active scalar. Nonlinearity. 7, 1495-1533 (1994). doi:10.1088/0951-7715/7/6/001

4. Constantin, P, Cordoba, D, Wu, J: On the critical dissipative quasi-geostrophic equation. Indiana Univ Math J. 50, 97-107 (2001)

5. Kiselev, A, Nazarov, F, Volberg, A: Global well-posedness for the critical 2D dissipative Quasi-geostrophic equation. Invent Math. 167, 445-453 (2007). doi:10.1007/s00222-006-0020-3

6. Khouider, B, Titi, ES: A Inviscid Regularization for the Surface Quasi-geostrophic Equ. Commun Pure Appl Math. 61, 1331-1346 (2008). doi:10.1002/cpa.20218

7. Chae, D: The quasi-geostrophic equation in the Triebel-Lizorkin spaces. Nonlinearity. 16, 479-495 (2003). doi:10.1088/ 0951-7715/16/2/307

8. Constantin, P, Wu, J: Behavior of solutions of 2D quasi-geostrophic equations. SIAM J Math Anal. 30, 937-948 (1999). doi:10.1137/S0036141098337333

9. $\mathrm{Wu}, \mathrm{J}$ : Inviscid limits and regularity estimates for the solution of the 2D dissipative Quasi-geostrophic equations. Indiana Univ Math J. 46, 1113-1124 (1997)

10. $W u$, J: Dissipative quasi-geostrophic equation with $L^{p}$ data. Electronic Journal of Differential Equations, Vol. 2001. 56, $1-13(2001)$

11. Wu, J: Quasi-geostrophic equation with initial data in Morrey spaces. Nonlinear Analysis. 10, 1409-1420 (1997)

12. Wu, J: Solutions of the 2D quasi-geostrophic equation in Hölder spaces. Nonlinearity. 62, 579-594 (2005)

13. Constantin, P, Córdoba, D, Wu, J: On the critical dissipative Quasi-geostrophic equation. Indiana Univ Math J. 50, 97-107 (2001)

14. Córdoba, A, Córdoba, D: A maximum principle applied to Quasi-geostrophic equations. Comm Math Phys. 249, 511-528 (2004)

15. Caffarelli, L, Vasseur, A: Drift diffusion equations with fractional diffusion and the Quasi-geostrophic equation. Ann Math. 171(3), 1903-1930 (2010). doi:10.4007/annals.2010.171.1903

16. Constantin, P, Wu, J: Regularity of Hölder continuous solutions of the supercritical quasi-geostrophic equation. Ann Inst H Poincare Anal Non Lineaire. 25(6), 1103-1110 (2008). doi:10.1016/j.anihpc.2007.10.001

17. Ju, N: The maximum principle and the global attractor for the dissipative Quasi-geostrophic equation. Comm Math Phys. 255, 161-181 (2005). doi:10.1007/s00220-004-1256-7 
18. Dong, H, Du, D: Global well-posedness and a decay estimate for the critical dissipative Quasi-geostrophic equation in the whole space. Discrete Contin Dyn Syst. 21(4), 1095-1101 (2008)

doi:10.1186/1687-2770-2011-41

Cite this article as: $\mathrm{Li}$ and Wang: Global existence for the regularized surface Quasi-geostrophic equation and its inviscid limits. Boundary Value Problems 2011 2011:41.

Submit your manuscript to a SpringerOpen ${ }^{\odot}$ journal and benefit from:

- Convenient online submission

- Rigorous peer review

- Immediate publication on acceptance

- Open access: articles freely available online

- High visibility within the field

- Retaining the copyright to your article

Submit your next manuscript at $\gg$ springeropen.com 